\title{
Concealment by uniform motion
}

\section{Tom G. Mackay \\ T.Mackay@ed.ac.uk}

\section{Akhlesh Lakhtakia \\ akhlesh@psu.edu}

School of Mathematics, James Clerk Maxwell Building, University of Edinburgh, Edinburgh EH9 3JZ, UK

CATMAS - Computational \& Theoretical Materials Sciences Group, Department of Engineering Science \& Mechanics, 212 Earth \& Engineering Sciences Building, Pennsylvania State University, University Park, PA, 16802-6812, USA

The perceived lateral position of a transmitted beam, upon propagating through a slab made of homogeneous, isotropic, dielectric material at an oblique angle, can be controlled through varying the velocity of the slab. In particular, on judiciously selecting the slab velocity, the transmitted beam can emerge from the slab with no lateral shift in position. Thereby, a degree of concealment can be achieved, as exemplified by numerical simulations involving a 2D Gaussian beam. [D0I: 10.2971/jeos.2007.07003]

Keywords: Minkowski constitutive relations, moving slab, Gaussian beam, counterposition

\section{Introd uction}

The topic of invisibility - which is a very old one in optics and electromagnetics [1] — has lately acquired a new lease of life with the advent of metamaterials [2,3]. In particular, the exotic electromagnetic possibilities offered by nonhomogeneous metamaterials may be harnessed to achieve cloaking, at least in principle [4]-[7]. The underlying theoretical arguments exploit the invariance of the Maxwell postulates under certain coordinate transformations.

A quite different approach to concealing a material is pursued in this paper. It is based on the perceived deflection of light by a material slab translating at constant velocity. No special material properties are required, but for illustrative purposes, we consider an isotropic, homogeneous, dielectric material. In two previous studies, we have demonstrated that the muchheralded negative-phase-velocity phenomenon often associated with negatively refracting electromagnetic metamaterials can be realized by conventional materials through the process of uniform motion $[8,9] .{ }^{1}$ Here, we demonstrate that a substantial degree of concealment may also be realized by uniform motion.

As regards notational matters, 3 vectors are in boldface, with the symbol identifying unit vectors. Double underlining signifies a $3 \times 3$ dyadic and $\underline{I}$ is the identity $3 \times 3$ dyadic. The superscript ${ }^{T}$ denotes the transpose of a column vector. The permittivity and permeability of vacuum are $\epsilon_{0}$ and $\mu_{0}$. The vacuum wavenumber is $k_{0}=\omega \sqrt{\epsilon_{0} \mu_{0}}$ with $\omega$ being the angular frequency, and the vacuum wavelength is $\lambda_{0}=2 \pi / k_{0}$.

\footnotetext{
${ }^{1}$ The phenomenon of negative refraction is not exclusively associated with metamaterials and relativisitic scenarios, as is demonstrated by the superposition eyes of certain moths and lobsters [10].
}

2 PLANEWAVE PROPAGATION INTO A UNIFORMLY MOVING H A L F-S P A C E

As a preliminary to concealment of a moving slab (section 3), let us consider a uniformly moving half-space. Suppose that a plane wave is launched with wavevector $\mathbf{k}_{i}=k_{i} \hat{\mathbf{k}}_{i}$ from vacuum $(z<0)$ towards the half-space $z>0$ occupied by an isotropic, nondissipative, dielectric material. This material moves at constant velocity $\mathbf{v}=v \hat{\mathbf{v}}=v \hat{\mathbf{x}}$, parallel to the interface and in the plane of incidence. In an inertial frame of reference that moves with the same velocity $\mathbf{v}$ with respect to the laboratory frame of reference wherein $\mathbf{k}_{i}$ is specified, the refracting material is characterized by relative permittivity $\epsilon_{r}$. The Minkowski constitutive relations of the moving halfspace in the laboratory frame of reference are [11]

$$
\left.\begin{array}{l}
\mathbf{D}(\mathbf{r})=\epsilon_{0} \epsilon_{r} \underline{\underline{\alpha}} \cdot \mathbf{E}(\mathbf{r})+\sqrt{\epsilon_{0} \mu_{0}}(\mathbf{m} \times \underline{\underline{I}}) \cdot \mathbf{H}(\mathbf{r}) \\
\mathbf{B}(\mathbf{r})=-\sqrt{\epsilon_{0} \mu_{0}}(\mathbf{m} \times \underline{\underline{I}}) \cdot \mathbf{E}(\mathbf{r})+\mu_{0} \underline{\underline{\alpha}} \cdot \mathbf{H}(\mathbf{r})
\end{array}\right\},
$$

where

$$
\left.\begin{array}{ll}
\underline{\underline{\alpha}}=\alpha \underline{\underline{I}}+(1-\alpha) \hat{\mathbf{v}} \hat{\mathbf{v}}, & \alpha=\frac{1-\beta^{2}}{1-\epsilon_{r} \beta^{2}}, \\
\mathbf{m}=m \hat{\mathbf{v}}, \quad m=\frac{\beta\left(\epsilon_{r}-1\right)}{1-\epsilon_{r} \beta^{2}}, \quad \beta=v \sqrt{\epsilon_{0} \mu_{0}}
\end{array}\right\} .
$$

In order to exclude the possibility of evanescent plane waves, $\epsilon_{r}>1$ is assumed. The envisaged scenario is illustrated schematically in Figure 1. 


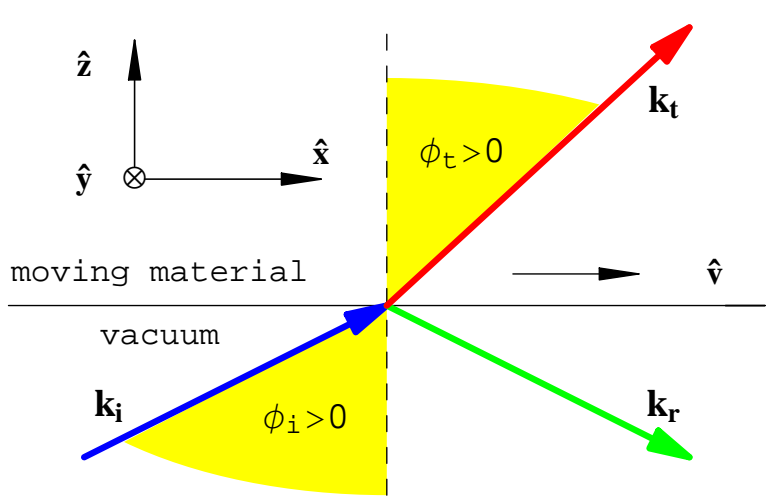

FIG. 1 A plane wave with wavevector $\mathbf{k}_{i}$ is incident from vacuum onto a half-space occupied by a simply moving dielectric material at an angle $\phi_{i}$ with respect to the unit vector $\hat{\mathbf{z}}$ normal to the planar interface. The moving material is characterized by relative permittivity $\epsilon_{r}>0$ in a co-moving frame of reference. As observed in the non-co-moving (laboratory) frame of reference wherein the incident plane wave is specified, the refracted wavevector $\mathbf{k}_{t}$ makes an angle $\phi_{t}$ with $\hat{\mathbf{z}}$.

The angle $\phi_{t}$ between the refracted wavevector $\mathbf{k}_{t}=k_{t} \hat{\mathbf{k}}_{t}$, as observed from the laboratory frame of reference, and the unit vector $\hat{\mathbf{z}}$ normal to the interface is related to the angle of incidence $\phi_{i}=\cos ^{-1}\left(\hat{\mathbf{k}}_{i} \cdot \hat{\mathbf{z}}\right)$ by [11]

$$
\phi_{t}=\sin ^{-1}\left(\frac{k_{0} \sin \phi_{i}}{k_{t}}\right)
$$

where

$$
k_{t}=k_{0}\left\{1+\xi\left[1-\beta\left(\hat{\mathbf{k}}_{i} \cdot \hat{\mathbf{v}}\right)\right]^{2}\right\}^{1 / 2}
$$

is the wavenumber of the refracted wave and $\xi=$ $\left(\epsilon_{r}-1\right) /\left(1-\beta^{2}\right)$. Since $0<\phi_{t}<\pi / 2 \forall \phi_{i} \in(0, \pi / 2)$, refraction is positive $\forall \beta \in(-1,1)$ [12].

The time-averaged Poynting vector of the refracted plane wave is given by [11]

$$
\begin{gathered}
\mathbf{P}_{t}=P_{t} \hat{\mathbf{P}}_{t}=\left(\left|C_{1}\right|^{2}+\epsilon_{r}\left|C_{2}\right|^{2}\right)\left(\mathbf{k}_{t} \times \hat{\mathbf{v}}\right)^{2}\left[\mathbf{k}_{t}+\right. \\
\left.+\xi \beta\left(k_{0}-\beta \mathbf{k}_{t} \cdot \hat{\mathbf{v}}\right) \hat{\mathbf{v}}\right],
\end{gathered}
$$

where $C_{1}$ and $C_{2}$ are constants, and the angle between $\hat{\mathbf{z}}$ and $\hat{\mathbf{P}}_{t}$ is

$$
\phi_{P}=\tan ^{-1}\left(\frac{\hat{\mathbf{P}}_{t} \cdot \mathbf{v}}{|v| \hat{\mathbf{P}}_{t} \cdot \hat{\mathbf{z}}}\right)
$$

As an illustrative example, the angle $\phi_{P}$ is plotted in Figure 2 against $\beta \in(-1,1)$ for $\phi_{i} \in\left\{15^{\circ}, 45^{\circ}, 75^{\circ}\right\}$, for the halfspace characterized by $\epsilon_{r}=6.0$. The orientation of the refracted time-averaged Poynting vector rotates towards the direction of motion as $\beta$ increases from -1 . The counterposition regime - which occurs where $\phi_{P}<0$ for $\phi_{t}>0-$ is discussed elsewhere $[12,13]$.

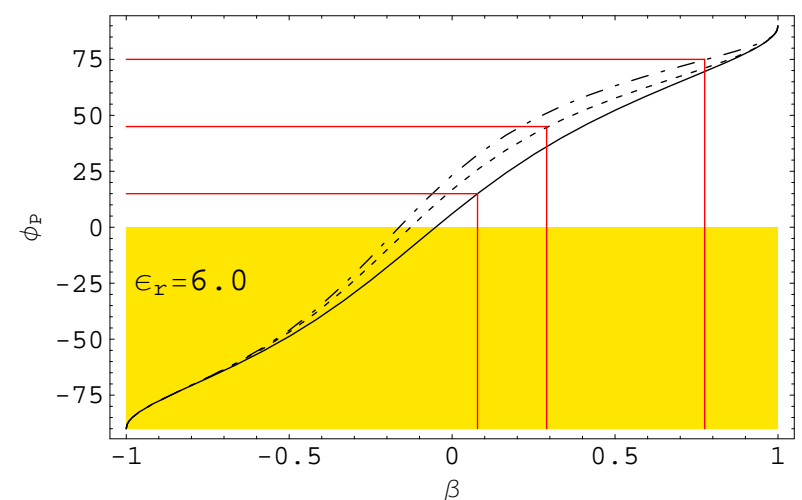

FIG. 2 The angle $\phi_{P}$ (in degree) between the time-averaged Poynting vector $\mathbf{P}_{t}$ and the unit vector $\hat{\mathbf{z}}$, plotted as a function of $\beta \in(-1,1)$, when the angle of incidence $\phi_{i}=15^{\circ}$ (solid curve), $45^{\circ}$ (dashed curve) and $75^{\circ}$ (broken dashed curve); and $\epsilon_{r}=6.0$. The red lines indicate where $\phi_{P}=\phi_{i}$. The counterposition regime $\left\{\phi_{P}<0^{\circ}, \phi_{t}>0^{\circ}\right\}$ is shaded.

In connection with Figure 2, it is of particular interest here that $\phi_{P}=\phi_{i}$ at (i) $\beta=0.08$ for $\phi_{i}=15^{\circ}$, (ii) $\beta=0.29$ for $\phi_{i}=45^{\circ}$, and (iii) $\beta=0.78$ for $\phi_{i}=75^{\circ}$. That is, there exist angles of incidence at which the time-averaged Poynting vector is not deflected by the uniformly moving half-space. This suggests that it may be possible for a light beam - not to be confused with a plane wave - to pass through a uniformly moving slab at an oblique angle without experiencing a lateral shift in position. That suggestion inspired the research presented in the next section.

\section{BEAM PROPAGATION THROUGH A UNIFORMLY MOVING SLAB}

Suppose that the uniformly moving half-space considered in section 2 is now replaced by a slab of thickness $L$ moving at constant velocity $\mathbf{v}=v \hat{\mathbf{x}}$ parallel to its two surfaces, as schematically illustrated in Figure 3. The slab - which is characterized, as before, by $\epsilon_{r}>1$ in a co-moving reference frame - is sandwiched by two vacuous half-spaces.

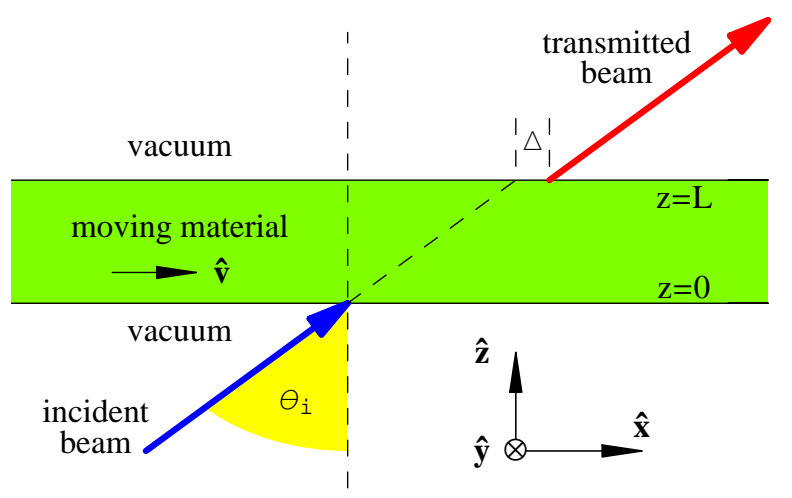

FIG. $3 \mathrm{~A}$ beam is incident onto a simply moving slab at an angle $\theta_{i}$ with respect to the unit vector $\hat{\mathbf{z}}$ normal to the planar interface. The moving material is characterized by relative permittivity $\epsilon_{r}>0$ in a co-moving frame of reference. As observed in the non-co-moving (laboratory) frame of reference wherein the incident plane wave is specified, the transmitted beam is shifted by $\Delta$, parallel to $\hat{\mathbf{x}}$, relative to its position if the slab were absent. 
A 2D beam with electric field phasor [14]

$$
\mathbf{E}_{i}(x, z)=\int_{-\infty}^{\infty} \mathbf{e}_{i}(\vartheta) \Psi(\vartheta) \exp \left[i\left(\mathbf{k}_{i} \cdot \mathbf{r}\right)\right] d \vartheta, z \leq 0,
$$

is incident upon the slab at a mean angle $\theta_{i}$ relative to the slab normal direction $\hat{z}$. The beam is represented as an angular spectrum of plane waves, with

$$
\begin{aligned}
\mathbf{k}_{i}=k_{0}\left[\left(\vartheta \cos \theta_{i}+\sqrt{1-\vartheta^{2}} \sin \theta_{i}\right) \hat{\mathbf{x}}-\right. \\
\left.\quad-\left(\vartheta \sin \theta_{i}-\sqrt{1-\vartheta^{2}} \cos \theta_{i}\right) \hat{\mathbf{z}}\right]
\end{aligned}
$$

being the wavevector of each planewave contributor. The angular-spectral function $\Psi(\vartheta)$ is taken to have the Gaussian form [14]

$$
\Psi(\vartheta)=\frac{k_{0} w_{0}}{\sqrt{2 \pi}} \exp \left[-\frac{\left(k_{0} w_{0} \vartheta\right)^{2}}{2}\right],
$$

with $w_{0}$ being the width of the beam waist. Two polarization states are considered: parallel to the plane of incidence, i.e.,

$$
\begin{aligned}
\mathbf{e}_{i}(\vartheta) \equiv \mathbf{e}_{\|}= & \left(\vartheta \sin \theta_{i}-\sqrt{1-\vartheta^{2}} \cos \theta_{i}\right) \hat{\mathbf{x}} \\
& +\left(\vartheta \cos \theta_{i}+\sqrt{1-\vartheta^{2}} \sin \theta_{i}\right) \hat{\mathbf{z}},
\end{aligned}
$$

and perpendicular to the plane of incidence, i.e.,

$$
\mathbf{e}_{i}(\vartheta) \equiv \mathbf{e}_{\perp}=\hat{\mathbf{y}}
$$

As the incident beam has the spatial Fourier representation (7), the reflected and the transmitted beams must also have similar representations. The electric field phasor of the reflected beam is given as

$$
\mathbf{E}_{r}(x, z)=\int_{-\infty}^{\infty} \mathbf{e}_{r}(\vartheta) \Psi(\vartheta) \exp \left[i\left(\mathbf{k}_{r} \cdot \mathbf{r}\right)\right] d \vartheta, \quad z \leq 0,
$$

with

$$
\begin{aligned}
\mathbf{k}_{r}=k_{0}\left[\left(\vartheta \cos \theta_{i}\right.\right. & \left.+\sqrt{1-\vartheta^{2}} \sin \theta_{i}\right) \hat{\mathbf{x}} \\
& \left.+\left(\vartheta \sin \theta_{i}-\sqrt{1-\vartheta^{2}} \cos \theta_{i}\right) \hat{\mathbf{z}}\right]
\end{aligned}
$$

and

$$
\mathbf{e}_{r}(\vartheta)=\left\{\begin{array}{c}
r_{\|}\left[-\left(\vartheta \sin \theta_{i}-\sqrt{1-\vartheta^{2}} \cos \theta_{i}\right) \hat{\mathbf{x}}\right. \\
\left.+\left(\vartheta \cos \theta_{i}+\sqrt{1-\vartheta^{2}} \sin \theta_{i}\right) \hat{\mathbf{z}}\right] \text { for } \mathbf{e}_{i}(\vartheta)=\mathbf{e}_{\|} \\
r_{\perp} \mathbf{e}_{\perp} \text { for } \mathbf{e}_{i}(\vartheta)=\mathbf{e}_{\perp}
\end{array} .\right.
$$

The electric field phasor of the transmitted beam is given as

$$
\mathbf{E}_{t}(x, z)=\int_{-\infty}^{\infty} \mathbf{e}_{t}(\vartheta) \Psi(\vartheta) \exp \left\{i\left[\mathbf{k}_{t} \cdot(\mathbf{r}-L \hat{\mathbf{z}})\right]\right\} d \vartheta, \quad z \geq L,
$$

with $\mathbf{k}_{t}=\mathbf{k}_{i}$ and

$$
\mathbf{e}_{t}(\vartheta)=\left\{\begin{array}{ccc}
t_{\|} \mathbf{e}_{i}(\vartheta) & \text { for } & \mathbf{e}_{i}(\vartheta)=\mathbf{e}_{\|} \\
t_{\perp} \mathbf{e}_{\perp} & \text { for } & \mathbf{e}_{i}(\vartheta)=\mathbf{e}_{\perp}
\end{array} .\right.
$$

Expressions for the reflection coefficients $r_{\|, \perp}$ and transmission coefficients $t_{\|, \perp}$ are provided in equations (A.16)-(A.19) in Appendix A.
In view of Figure 2, we fixed the mean angle of incidence of the beam at $\theta_{i}=45^{\circ}$ and explored the behaviour of the transmitted beam for $\beta<0.29, \beta=0.29$ and $\beta>0.29$. The energy density of the beam in both half-spaces, as measured by

$$
|\mathbf{E}|^{2}=\left\{\begin{array}{ll}
\left|\mathbf{E}_{i}+\mathbf{E}_{r}\right|^{2} & \text { for } \quad z \leq 0 \\
\left|\mathbf{E}_{t}\right|^{2} & \text { for } z \geq L
\end{array},\right.
$$

is mapped for $z / \lambda_{0} \in(-8,12)$ and $x / \lambda_{0} \in(-25,25)$ in Figure 4 with the slab thickness $L=4 \lambda_{0}$. The restriction $\vartheta \in[-1,1]$ was imposed to exclude evanescence. A beam waist of $w_{0}=1.75 \lambda_{0}$ was selected for all calculations. We considered $\beta \in\{-0.15,0.29,0.8\}$ for both $\mathbf{e}_{i}=\mathbf{e}_{\|}$and $\mathbf{e}_{i}=\mathbf{e}_{\perp}$. The numerical values for the beam waist and slab thickness were chosen in order to accentuate the clarity of Figure 4.
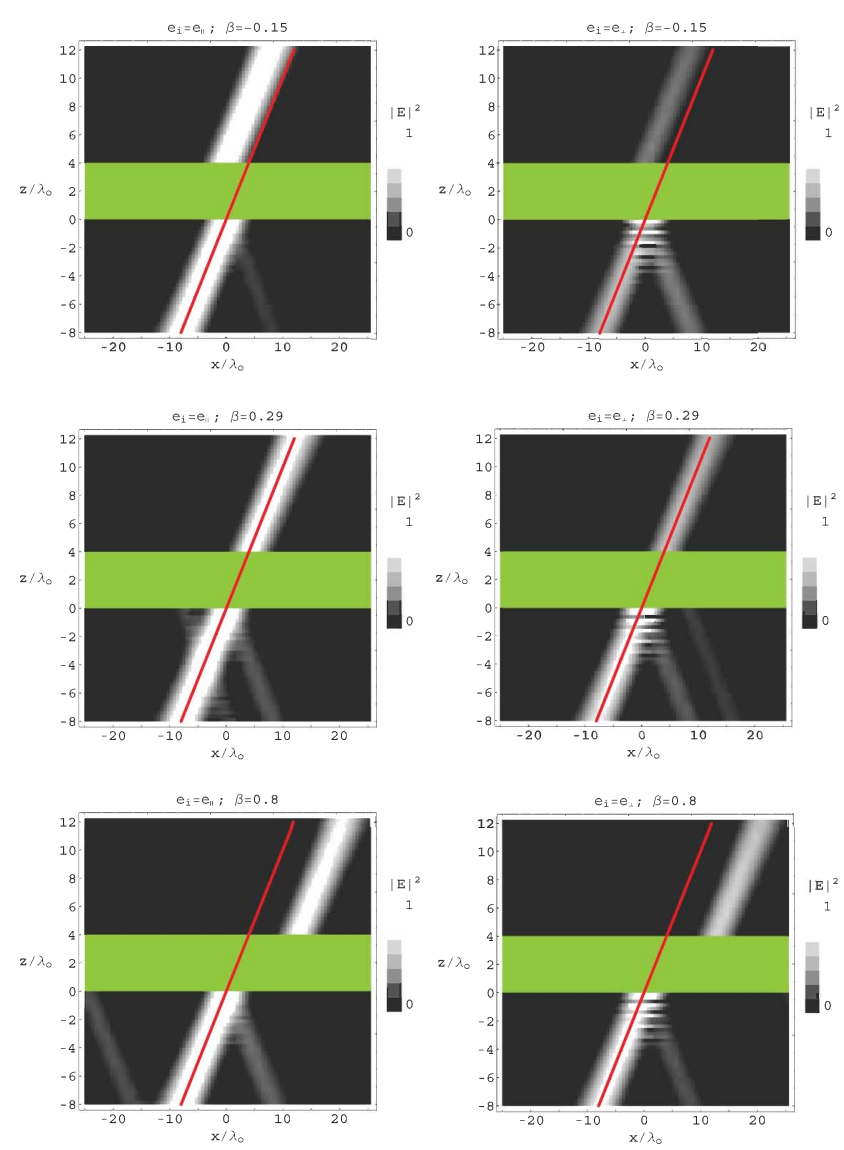

FIC. 4 Normalized $|\mathbf{E}|^{2}$ is mapped in the $x z$ plane for a 2D Gaussian beam incident onto a simply moving slab at an angle $\theta_{i}=45^{\circ}$. The relative speed of the slab is: $\beta=-0.15$ (top); $\beta=0.29$ (middle); and $\beta=0.8$ (bottom). The electric field phasor of the incident beam is polarized parallel (left) and perpendicular (right) to the plane of incidence. The red line indicates the mean beam position in the absence of the moving slab.

The fringes which can observed in Figure 4 are artefacts. These are due to aliasing errors associated with the discretization of the spatial Fourier representation of the beam and the finite integration domain used for the computations [15]. The apparent secondary reflected beams which lie to the left of the incident beam in Figure 4 for $\mathbf{e}_{i}=\mathbf{e}_{\|}$with $\beta=0.29$ and $\beta=0.8$ are similarly artefacts due to aliasing errors. 
Regardless of the polarization state, the transmitted beam does not undergo a lateral shift (relative to the incident beam) when $\beta=0.29$. However, the transmitted beam is laterally shifted in the direction of $-\hat{\mathbf{x}}$ when $\beta<0.29$ and in the direction of $+\hat{\mathbf{x}}$ when $\beta>0.29$. The energy densities of the reflected and transmitted beams are sensitive to $\beta$ and the polarization state of the incident beam.

A more quantitative representation of the transmitted beam is provided in Figure 5, wherein $|\mathbf{E}|^{2}$ is plotted against $x$ for $\beta \in\{-0.15,0.29,0.8\}$ at $z=4 \lambda_{0}$. For comparison, $|\mathbf{E}|^{2}$ for the beam in the absence of the moving slab is also plotted. It is clear that the beam position for $\beta=0.29$ coincides with the beam position in the absence of the moving slab. At $\beta=0.29$, the peak energy density of the transmitted beam for the case of parallel polarization is approximately $11 \%$ less than it would be if the slab were absent; the corresponding figure for perpendicular polarization is $38 \%$. The energy losses in transmission presented here are typical rather than minimal. The transmission losses could be reduced by adjusting the beam parameters, but there will always be some loss.
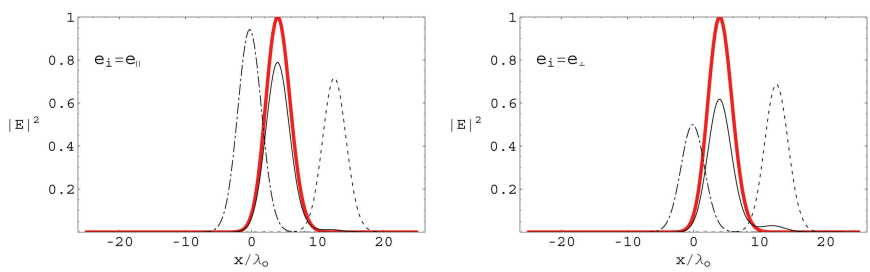

FIG. 5 Normalized $|\mathbf{E}|^{2}$ at $z=4 \lambda_{0}$ for $\beta=-0.15$ (broken dashed curve); $\beta=0.29$ (solid dark curve); and $\beta=0.8$ (dashed curve). The solid red curve represents the normalized $|\mathbf{E}|^{2}$ in the absence of the moving slab. The electric field phasor of the incident beam is polarized parallel (left) and perpendicular (right) relative to the plane of incidence.

The median shift of transmitted beam in relation to the incident beam is defined as

$$
\begin{aligned}
\Delta= & \left(\int_{-\infty}^{\infty} x\left|\mathbf{E}_{t}(x, L)\right|^{2} d x\right)\left(\int_{-\infty}^{\infty}\left|\mathbf{E}_{t}(x, L)\right|^{2} d x\right)^{-1} \\
& -\left(\int_{-\infty}^{\infty} x\left|\mathbf{E}_{i}(x, L)\right|^{2} d x\right)\left(\int_{-\infty}^{\infty}\left|\mathbf{E}_{i}(x, L)\right|^{2} d x\right)^{-1}
\end{aligned}
$$

For both parallel and perpendicular polarizations, $\Delta$ is plotted against $\beta \in(-1,1)$ in Figure 6.
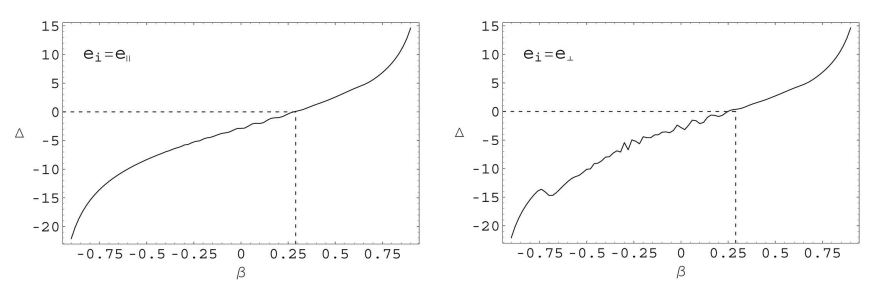

FIG. 6 The median beam shift $\Delta$ at $z=4 \lambda_{0}$, plotted against $\beta$. The electric field phasor of the incident beam is polarized parallel (left) and perpendicular (right) relative to the plane of incidence. Zero median beam shift at $\beta=0.29$ is indicated by dashed lines.
Thus, regardless of the polarization state, the beam can be shifted laterally along $\pm \hat{\mathbf{x}}$ by means of uniform motion. In particular, the zero beam shift at $\beta=0.29$ is further confirmed in Figure 6 . The wrinkles apparent in the graphs of Figure 6 are an artefact of the computations.

\section{C O N C L U D IN G R E M A R K S}

Our numerical investigations show that a $2 \mathrm{D}$ beam can pass obliquely through a uniformly moving slab without undergoing a lateral shift in its position. At a fixed angle of beam incidence, this effect occurs only for a unique translational slab velocity. However, extrapolating from Figure 2, for every angle of beam incidence a slab velocity can be found at which the beam undergoes no lateral shift. Furthermore, the zero lateral deflection results presented in Figure 4 will also hold for a pulsed beam at a fixed angle of incidence, provided that the constitutive parameters of the moving slab do not vary with angular frequency in the pulse spectrum. This finding is in agreement with the well-known Fresnel drag [11].

Concealment achieved by uniform motion - as indicated by the absence of a lateral shift - is not $100 \%$ perfect due to reflections but, in the particular case of the example considered in section 3, almost $90 \%$ of the peak energy density of the beam can be transmitted without deflection. Other schemes for concealment have deficiencies too: most notably, highly specialized, nonhomogeneous metamaterials are required and the degree of concealment is not perfect [4]. Our results indicate that the effectiveness of a cloaking device could be enhanced by motion, particularly for future applications in space.

\section{A C K N O W L E D G M E T}

TGM is supported by a Royal Society of Edinburgh/Scottish Executive Support Research Fellowship.

\section{A A P P E N DIX}

The reflection coefficients $r_{\|, \perp}$ and transmission coefficients $t_{\|, \perp}$ are straightforwardly calculated by solving the reflectiontransmission problem as a boundary value problem. We outline the procedure here, further details being available elsewhere [16].

Consider the plane wave with electric and magnetic field phasors

$$
\left.\begin{array}{l}
\mathbf{E}(x, z)=\tilde{\mathbf{e}}(z, \theta) \exp \left(i k_{0} x \sin \theta\right) \\
\mathbf{H}(x, z)=\tilde{\mathbf{h}}(z, \theta) \exp \left(i k_{0} x \sin \theta\right)
\end{array}\right\}
$$

propagating in the $x z$ plane. As in section 3, a moving slab described by the Minkowski constitutive relations (1) occupies the region between $z=0$ and $z=L$; elsewhere there is vacuum. We write

$$
\tilde{\mathbf{p}}(z, \theta)=\tilde{p}_{x}(z, \theta) \hat{\mathbf{x}}+\tilde{p}_{y}(z, \theta) \hat{\mathbf{y}}+\tilde{p}_{z}(z, \theta) \hat{\mathbf{z}}, \quad(p=e, h)
$$


Substitution of equations (1) and (A.1) into the source-free Maxwell curl postulates

$$
\begin{aligned}
& \nabla \times \mathbf{E}(x, z)-i \omega \mathbf{B}(x, z)=\mathbf{0}, \\
& \nabla \times \mathbf{H}(x, z)+i \omega \mathbf{D}(x, z)=\mathbf{0},
\end{aligned}
$$

delivers four differential equations and two algebraic equations. The latter two equations are easily solved for $\tilde{e}_{z}$ and $\tilde{h}_{z}$. Thereby, the four differential equations may be expressed in matrix form as

$$
\frac{\partial}{\partial z}[\mathbf{f}(z, \theta)]=i k_{0}[\mathbf{P}(\theta)][\mathbf{f}(z, \theta)]
$$

where

$$
[\mathbf{f}(z, \theta)]=\left[\tilde{e}_{x}(z, \theta), \tilde{e}_{y}(z, \theta), \tilde{h}_{x}(z, \theta), \tilde{h}_{y}(z, \theta)\right]^{T}
$$

is a column vector and

$$
\mathbf{P}(\theta)=\left[\begin{array}{cccc}
0 & 0 & 0 & \eta_{0} \rho \\
0 & 0 & -\eta_{0} & 0 \\
0 & -\epsilon_{r} \rho / \eta_{0} & 0 & 0 \\
\epsilon_{r} / \eta_{0} & 0 & 0 & 0
\end{array}\right]
$$

is a $4 \times 4$ matrix with

$$
\rho=\alpha-\frac{(m+\sin \theta)^{2}}{\epsilon_{r} \alpha} .
$$

The solution to (A.5) is conveniently expressed as

$$
[\mathbf{f}(L, \theta)]=[\mathbf{M}(L, \theta)][\mathbf{f}(0, \theta)],
$$

in terms of the transfer matrix

$$
[\mathbf{M}(L, \theta)]=\exp \left\{i k_{0}[\mathbf{P}(\theta)] L\right\} .
$$

Now we turn to the incident, reflected and transmitted plane waves. Let the incident plane wave be represented in terms of linear polarization components as

$$
\left.\begin{array}{c}
\tilde{\mathbf{e}}_{i}(z, \theta)=\left[a_{\perp} \hat{\mathbf{y}}+a_{\|}(-\cos \theta \hat{\mathbf{x}}+\sin \theta \hat{\mathbf{z}})\right] \times \\
\exp \left(i k_{0} z \cos \theta\right) \\
\tilde{\mathbf{h}}_{i}(z, \theta)=\eta_{0}^{-1}\left[a_{\perp}(-\cos \theta \hat{\mathbf{x}}+\sin \theta \hat{\mathbf{z}})-a_{\|} \hat{\mathbf{y}}\right] \times \\
\exp \left(i k_{0} z \cos \theta\right)
\end{array}\right\}, z \leq 0 .
$$

The corresponding reflected and transmitted plane waves are given as

$$
\left.\begin{array}{c}
\tilde{\mathbf{e}}_{r}(z, \theta)=\left[a_{\perp} r_{\perp} \hat{\mathbf{y}}+a_{\|} r_{\|}(\cos \theta \hat{\mathbf{x}}+\sin \theta \hat{\mathbf{z}})\right] \times \\
\exp \left(-i k_{0} z \cos \theta\right) \\
\tilde{\mathbf{h}}_{r}(z, \theta)=\eta_{0}^{-1}\left[a_{\perp} r_{\perp}(\cos \theta \hat{\mathbf{x}}+\sin \theta \hat{\mathbf{z}})-a_{\|} r_{\|} \hat{\mathbf{y}}\right] \times \\
\exp \left(-i k_{0} z \cos \theta\right)
\end{array}\right\}, z \leq 0
$$

and

$$
\left.\begin{array}{c}
\tilde{\mathbf{e}}_{t}(z, \theta)=\left[a_{\perp} t_{\perp} \hat{\mathbf{y}}+a_{\|} t_{\|}(-\cos \theta \hat{\mathbf{x}}+\sin \theta \hat{\mathbf{z}})\right] \times \\
\exp \left[i k_{0}(z-L) \cos \theta\right] \\
\tilde{\mathbf{h}}_{t}(z, \theta)=\eta_{0}^{-1}\left[a_{\perp} t_{\perp}(-\cos \theta \hat{\mathbf{x}}+\sin \theta \hat{\mathbf{z}})-a_{\|} t_{\|} \hat{\mathbf{y}}\right] \times \\
\exp \left[i k_{0}(z-L) \cos \theta\right]
\end{array}\right\}, z \geq L,
$$

respectively. By application of the boundary conditions at $z=0$ and $z=L$ to the solution (A.9), the reflection and transmission coefficients are found to be related by the matrix algebraic equation

$$
[\mathbf{K}(\theta)]\left[t_{\perp}, t_{\|}, 0,0\right]^{T}=[\mathbf{M}(L, \theta)][\mathbf{K}(\theta)]\left[1,1, r_{\perp}, r_{\|}\right]^{T},
$$

wherein

$$
\mathbf{K}(\theta)=\left[\begin{array}{cccc}
0 & -\cos \theta & 0 & \cos \theta \\
1 & 0 & 1 & 0 \\
-\eta_{0}^{-1} \cos \theta & 0 & \eta_{0}^{-1} \cos \theta & 0 \\
0 & -\eta_{0}^{-1} & 0 & -\eta_{0}^{-1}
\end{array}\right] .
$$

Thus, after some manipulation, the reflection and transmission coefficients emerge as

$r_{\perp}=\frac{\left(\cos ^{2} \theta-\epsilon_{r} \rho\right) \sin \left(k_{0} L \sqrt{\epsilon_{r} \rho}\right)}{\left(\cos ^{2} \theta+\epsilon_{r} \rho\right) \sin \left(k_{0} L \sqrt{\epsilon_{r} \rho}\right)+2 i \sqrt{\epsilon_{r} \rho} \cos \left(k_{0} L \sqrt{\epsilon_{r} \rho}\right) \cos \theta}$

$r_{\|}=\frac{\left(\rho-\epsilon_{r} \cos ^{2} \theta\right) \sin \left(k_{0} L \sqrt{\epsilon_{r} \rho}\right)}{\left(\epsilon_{r} \cos ^{2} \theta+\rho\right) \sin \left(k_{0} L \sqrt{\epsilon_{r} \rho}\right)+2 i \sqrt{\epsilon_{r} \rho} \cos \left(k_{0} L \sqrt{\epsilon_{r} \rho}\right) \cos \theta}$,

$t_{\perp}=\frac{-2 i \sqrt{\epsilon_{r} \rho} \cos \theta}{\left(\cos ^{2} \theta+\epsilon_{r} \rho\right) \sin \left(k_{0} L \sqrt{\epsilon_{r} \rho}\right)+2 i \sqrt{\epsilon_{r} \rho} \cos \left(k_{0} L \sqrt{\epsilon_{r} \rho}\right) \cos \theta}$,

$$
t_{\|}=\frac{2 i \sqrt{\epsilon_{r} \rho} \cos \theta}{\left(\epsilon_{r} \cos ^{2} \theta+\rho\right) \sin \left(k_{0} L \sqrt{\epsilon_{r} \rho}\right)+2 i \sqrt{\epsilon_{r} \rho} \cos \left(k_{0} L \sqrt{\epsilon_{r} \rho}\right) \cos \theta} .
$$

\section{References}

[1] E. Wolf and T. Habashy, "Invisible bodies and uniqueness of the inverse scattering problem" J. Mod. Optics 40, 785-792 (1993).

[2] V. A. Fedotov, P.L. Mladyonov, S.L. Prosvirnin, and N.I. Zheludev, "Planar electromagnetic metamaterial with a fish scale structure" Phys. Rev. E 72, 056613 (2005).

[3] A. Alù and N. Engheta, "Achieving transparency with plasmonic and metamaterial coatings" Phys. Rev. E 72, 016623 (2005). Erratum 73, 019906(E) (2006).

[4] J. Hecht, "Photonic frontiers: metamaterials" Laser Focus World (July 2006). http://lfw.pennnet.com/articles/articledisplay.cfm?article-id=259931

[5] U. Leonhardt, "Optical conformal mapping" Science 312, 1777-1780 (2006).

[6] G. W. Milton and N-A. P. Nicorovici, "On the cloaking effects associated with anomalous localized resonance" Proc. R. Soc. Lond. A 462, 3027-3059 (2006).

[7] D. Schurig, J.J. Mock, B.J. Justice, S.A. Cummer, J.B. Pendry, A.F. Starr, and D. R. Smith, "Metamaterial electromagnetic cloak at microwave frequencies" Science 314, 977-980 (2006).

[8] T. G. Mackay and A. Lakhtakia, "Negative phase velocity in a uniformly moving, homogeneous, isotropic, dielectric-magnetic medium" J. Phys. A: Math. Gen. 37, 5697-5711 (2004).

[9] T. G. Mackay and A. Lakhtakia, "On electromagnetics of an isotropic chiral medium moving at constant velocity" Proc. R. Soc. Lond. A 463, 397-418 (2007). 
[10] D.G. Stavenga, "Invertebrate superposition eyes - structures that behave like metamaterial with negative refractive index" J. Eur. Opt. Soc - Rapid Pub. 1, 06010 (2006).

[11] H. C. Chen, Theory of electromagnetic waves (McGraw-Hill, New York, NY, USA, 1983), Chap. 8.

[12] T.C. Mackay and A. Lakhtakia, “Counterposition and negative refraction due to uniform motion" Microwave Opt. Technol. Lett. (to appear). http://arxiv.org/abs/physics/0610039

[13] A. Lakhtakia and M. W. McCall, “Counterposed phase velocity and energy-transport velocity vectors in a dielectric-magnetic uniaxial medium" Optik 115, 28-30 (2004).

[14] H. A. Haus, Waves and fields in optoelectronics (Prentice-Hall, Englewood Cliffs, NJ, USA, 1984).

[15] S. W. Smith, The scientist and engineer's guide to digital signal processing (California Technical Publishing, San Diego, CA, USA, 1997).

[16] A. Lakhtakia and R. Messier, Sculptured thin films: Nanoengineered optics and morphology (SPIE Press, Bellingham, WA, USA, 2005). 